Voix et Images

voixetimages

\title{
Angoisses métisses
}

\section{François Paré}

Volume 29, numéro 1 (85), automne 2003

\section{Claire Martin}

URI : https://id.erudit.org/iderudit/007544ar

DOI : https://doi.org/10.7202/007544ar

Aller au sommaire du numéro

\section{Éditeur(s)}

Université du Québec à Montréal

\section{ISSN}

0318-9201 (imprimé)

1705-933X (numérique)

Découvrir la revue

Citer ce compte rendu

Paré, F. (2003). Compte rendu de [Angoisses métisses]. Voix et Images, 29(1), 131-135. https://doi.org/10.7202/007544ar d'utilisation que vous pouvez consulter en ligne.

https://apropos.erudit.org/fr/usagers/politique-dutilisation/ 


\author{
ES S A I / É T U D E S
}

Angoisses métisses

$+++$

FRANÇOIS PARÉ

Université de Waterloo

J'ai acheté le petit livre de Giorgio Agamben dans une librairie de Montréal, l'été dernier. Je n'en connaissais rien au moment de l'achat, n'ayant alors lu d'Agamben que des extraits épars, traduits en anglais. Je ne me doutais pas que ce petit opuscule jouerait, quelques semaines plus tard, un rôle central dans l'élaboration de cette chronique. Comme d'autres, j'étais frappé, troublé même, par l'assurance presque théâtrale des propos d'Agamben. N'y avait-il donc aucune part d'incertitude dans son diagnostic de l'Occident malade? Comment pouvait-il être si sûr, lui qui notait justement l'épuisement des certitudes idéologiques?

Moyens sans fins rassemble une dizaine d'essais journalistiques, traitant de divers sujets, dont l'affaiblissement des nationalismes et la montée d'une société consommatrice, productrice de vacuité et d'apparence. Pour Agamben, l'Occident, soumis au morcellement des grands mythes fédérateurs, vit depuis la Seconde Guerre mondiale l'évidement paroxystique du sens commun et la dictature d'une multitude d'états d'exception, privant de fondement toute éthique du partage : «Tandis que le déclin de l'État laisse partout subsister son enveloppe vide, pure structure de souveraineté et de domination, la société dans son ensemble est par contre irrévocablement vouée au modèle de la société de consommation et de production en vue du seul bienêtre $^{1}$.» Si ce constat d'échec est cinglant, il n'est pas sans appel. Agamben fait miroiter le surgissement de nouveaux paradigmes identitaires, issus des décombres de l'ancien monde.

Il faut dire que j'admire énormément l'aplomb du philosophe de la «vie nue». Agamben joue le grand jeu devant son lecteur et se livre à de véritables envolées prophétiques, dans lesquelles paraît s'énoncer notre avenir à tous sur cette terre. Comme le joueur de football professionnel, feignant la douleur devant l'arbitre médusé, l'écriture du penseur politique ne semble dénoncer le déclin généralisé des discours universels que pour se positionner à son tour dans le champ de la philosophie humaniste de l'après-guerre en Europe. C'est ainsi que, pour Agamben, la succession des crises qui étranglent la conscience européenne depuis le milieu du siècle dernier se reporte naturellement sur l'ensemble de l'humanité, comme si la décomposition violente des États et

$$
++
$$

1 Giorgio Agamben, Moyens sans fins. Notes sur la politique, Paris, Rivages Poche, 2002 [1995], p. 126. 
des dictatures nationales sur le Vieux-Continent autorisait la pensée à s'approprier à nouveau, sans aucune espèce de scrupule, les modes de représentation de la vérité. Ce curieux paradoxe et le malaise qu'il engendre chez moi, malgré mon affection pour l'écriture scintillante d'Agamben, ont accompagné ma lecture des deux ouvrages, en apparence fort différents, qui sont l'objet de mon attention dans cette chronique. Le premier porte sur la notion de communauté métisse, tandis que le second retrace l'histoire assez compliquée, à vrai dire, de la notion de plagiat.

Si j'ai tant insisté sur l'essai de Giorgio Agamben, c'est que ce penseur est souvent cité par les collaborateurs et collaboratrices du recueil collectif, s'intitulant Politique de la parole, paru sous la direction de Pierre Ouellet ${ }^{2}$. Issu d'un colloque tenu à l'Université du Québec à Montréal au lendemain des attentats du 11 septembre 2001, ce livre important est profondément marqué par certaines notions-phares de la pensée québécoise contemporaine, qui prennent dans le contexte des actes terroristes récents une dimension nouvelle. Dans son introduction, Pierre Ouellet rappelle, à la manière de Charles Taylor, que l'émergence de la «relation intersubjective» et le nivellement des rapports hiérarchiques d'appartenance dans la société occidentale annoncent l'avènement de modes de "cohabitation", par lesquels l'intégrité du sujet individuel repose désormais sur ses liens constitutifs avec les autres sujets. Politique de la parole, écrit Ouellet, cherche à comprendre ce nouvel agora, au moment où des événements d'une rare violence semblent en contredire la validité épistémologique. Que faire devant la destruction provoquée par les attentats du 11 septembre, dans cet espace du World Trade Center qui n'était absolument pas un espace européen, mais bien l'expression d'une autre forme, américaine cette fois, de prétention à l'universel? À lire l'ensemble des études qui nous sont proposées ici, on sent l'impuissance de l'écriture à rendre compte d'actes qui relèvent d'abjections jusque-là imprévues. Chez certains auteurs, le constat déclenche un pressant appel à mettre fin à la prolifération des «minitotalitarismes d'appartenance ", selon l'expression de Marc Angenot. Chez ce dernier, le «réenchantement du monde» ne peut découler des «tribalismes de ressentiment» qui, profitant du déclin généralisé des systèmes de pensée, s'acharnent à imposer leur «absolutisme culturel» (48-49). Pour Angenot, comme, du reste, pour Régine Robin et Jacques Rancière, la mondialisation des échanges économiques ne se laisse jamais saisir comme une manifestation plus diffuse des anciens pouvoirs hégémoniques. Cette occidentalisation des cultures humaines est-elle aussi bienveillante qu'on le suppose? Est-il possible que, loin de percevoir la vacuité de l'Occident, les auteurs des attentats terroristes aient au contraire suffoqué sous l'expansionnisme d'une dictature sans limite de cette vacuité? Si, comme le souligne June Nash dans un article récent, la multiplication apparente des réseaux de communication et des programmes de développement, mis de l'avant par les États de l'Amérique et de l'Europe, n'était que le masque d'une nouvelle commodification des rapports humains, on comprendrait plus facilement la rancœur qui anime aujourd'hui une partie de l'humanité contre la pensée occidentale et ses fantasmes d'ouverture à l'autre ${ }^{3}$.

Dans un essai intitulé «Vers les communautés technoculturelles: traitement de l'expérience et nouvelles visualisations», Michaël La Chance élabore une critique en 
règle du mensonge qui, selon lui, traverse de part en part les discours occidentaux. La Chance rejette avec véhémence le «scintillement de la sphère techno-magnétique» (77). La culture dans laquelle nous vivons ne peut entraîner qu'une aliénation irrémissible des rapports interpersonnels et interculturels. Or c'est justement cette aliénation qui rend impossible, tout au moins dans la sphère culturelle de l'Amérique, la compréhension de l'Autre en tant que différence. S'opposant à la reproduction mécanique des individualités, La Chance propose une théorie de la variation dont on verra toute l'importance dans les derniers paragraphes de cette chronique: "La machine semble garantir la transmission intégrale et une diffusion de copies rigoureusement identiques. Tandis que le vivant et le culturel introduisent toujours des variants» (85-86). De tels propos, repris sous d'autres formes dans divers articles de Politique de la parole, illustrent l'approche particulière d'une bonne part de la pensée québécoise récente sur les notions de communauté et de pluralité.

En effet, chez Pierre Ouellet, comme chez Simon Harel, Catherine Mavrikakis et Michaël La Chance, entre autres, le déclin généralisé des discours idéologiques, relevé par Giorgio Agamben, ne peut être compensé par la simple improvisation de réseaux individuels, informatiques ou autres, érigés en communautés transitoires. L'éclatement des communautés traditionnelles ne saurait, dans ce cas, enrayer la cohésion des formes culturelles de l'appartenance. Ce sont ces formes, difficiles à saisir dans leurs multiples différences, qui permettent, en revanche, de comprendre la violence encore présente des hégémonies de toutes sortes, qu'elles soient issues des colonialismes ou des impérialismes. Il n'est pas étonnant que, renonçant au modèle européen de la communauté de jeu ou de la communauté esthétique, telle qu'elle se trouve dans les écrits de Jacques Rancière, plusieurs des intellectuels québécois représentés ici cherchent à maintenir ce que Gad Soussana appelle la «communauté événementielle», susceptible de rendre compte de phénomènes réels, de cette «implosion, venue au sensible, de la communauté» (191, en italiques dans le texte). Ainsi, il devrait être possible de concevoir l'épuisement des systèmes traditionnels comme une mise en scène de la différence, de la variance, garantie par la culture elle-même.

Bien qu'elle soit souvent mentionnée dans Politique de la parole, la pensée de Giorgio Agamben restera toujours aveugle au surgissement de la violence terroriste. Très lié aux milieux américains, Agamben ne voit guère l'émergence d'un nouvel ordre colonial, pourtant très clair pour ceux qui vivent dans le sillage immédiat de sa violence. Ce qui est proprement terrifiant, c'est que la vacuité des systèmes idéologiques de l'Occident s'impose à son tour comme un impérialisme aussi «transparent " qu'intolérable. L'ouvrage dirigé par Pierre Ouellet ne rend pas entièrement compte de ces phénomènes "sensibles», encore très proches de nous; néanmoins, il témoigne amplement de la richesse de la pensée québécoise actuelle sur les concepts-clés de nation, de métissage culturel, de communauté et de droits de la personne.

L'envergure de l'ouvrage d'Yzabelle Martineau sur l'intertextualité et le plagiat semble au premier regard beaucoup plus limitée ${ }^{4}$. Mais la lecture de cette étude fort perspicace en révèle, au contraire, toute la pertinence théorique. Le faux littéraire retrace l'histoire du plagiat, depuis ses origines dans les «pratiques littéraires ambiguës» au Moyen Âge jusqu'aux conceptions plus modernes du droit d'auteur et de l'intertextualité.

$+++$

4 Yzabelle Martineau, Le faux littêraire. Plagiat littéraire, intertextualité et dialogisme, Québec, Nota bene, 2002, 284 p. 
Ce qui est intéressant dans l'approche adoptée par Martineau, c'est qu'elle déstabilise les perceptions juridiques qui entourent le plagiat, car celles-ci reposent sur une codification, au cœur même des cultures de l'Occident, de la singularité des œuvres et des concepts plus vastes de mixité, de variance et de pureté. Dès la Renaissance, au moment où se développe le livre imprimé, on assiste à ce que Martineau appelle "un nouvel ordre esthétique qui est celui de l'originalité» (17). L'objet-livre se présente alors comme le produit exclusif d'un seul auteur, dont la personne juridique et économique est graduellement reconnue par le pouvoir politique. L'histoire du plagiat gravite autour de cet important changement de paradigme.

Tout revient, écrit Martineau, à la conception que nous nous faisons de la littérature. Indistincte du discours social et donc profondément métissée, celle-ci pourrait être un bricolage d'emprunts et de citations. Dans ce cas, l'œuvre littéraire s'éloignerait assez radicalement de tout postulat d'originalité, puisqu'elle serait nourrie par une pratique généralisée, bien qu'inconsciente, du plagiat. À l'inverse, on admettra aisément que la même œuvre puisse justement refléter l'individualité de l'écrivain devant la norme, sa ferme résistance à l'envahissement d'un discours social, voué à la répétition et à la duplication. L'objectif de Martineau est de suivre à la trace un certain nombre d'ouvrages, souvent marginaux en leur temps, dans lesquels se déploie une véritable conception théorique des pratiques plagiaires. Il s'agit donc moins de faire l'histoire de la codification juridique de la singularité textuelle que de mettre en jeu des pratiques transgressives qui accompagnent fidèlement, depuis ses commencements, l'histoire du livre imprimé. Ce parcours est d'autant plus intéressant qu'il interroge le rôle que joue depuis longtemps l'université dans la mise en scène de la "pureté» des textes. Martineau note qu'en dépit de l'apparition récente des concepts plus ambigus de métissage et d'intertextualité en littérature, le milieu universitaire, surtout en Amérique du Nord, continue de démontrer un acharnement obsessionnel à traquer les plagiaires et à contrer toutes les formes de la duplication.

Le faux littéraire consacre de nombreuses pages à certains écrivains célèbres, victimes de diverses accusations de plagiat. Parmi ceux-là, il est sûr que Pierre Corneille se situe, comme l'écrit Martineau, à la charnière entre deux conceptions de l'emprunt et de l'imitation: "celle du poète, du lettré, que ne protégeait encore aucune loi, vendant ses services aux nobles dont il chantait les louanges et recevait en échange sustentation, logis, réputation, et celle de l'écrivain de profession, relativement libre, gérant lui-même son écriture, ses profits et même son avenir» (126). Mais Corneille était l'héritier d'une longue tradition française de l'emprunt, dont les bases se trouvent chez Joachim du Bellay et chez Michel de Montaigne. Martineau fait remarquer à juste titre que le traitement du texte métis, fortement nourri par la citation et l'imitation, varie beaucoup d'une sphère culturelle à l'autre. Dans la tradition française, le plagiat semble non seulement toléré, mais considéré comme une forme à part entière du savoir. Les cultures anglophones ont, au contraire, rapidement interdit et marginalisé les pratiques plagiaires.

Les conclusions de Martineau restent nuancées. Elles nous intéressent parce qu'elles ramènent la question du plagiat à ses ancrages dans la gestion culturelle de l'hétérogène et de la variance: «Le fait de reconnaître l'hétérogénéité, le métissage culturel, le vaste travail intertextuel qu'est la conception de tout texte [y compris, je dois le dire, cette chronique!] ne nous affranchit pas de nos fantasmes d'originalité» (231). En effet, symptôme de nos angoisses métisses, le plagiat nous permet de comprendre les liens étroits entre le livre imprimé et la recherche de la singularité. Mais le livre est du 
même souffle le lieu où se manifeste en priorité l'utopie du plagiat généralisé. Une pensée de la variance, à la manière de Michaël La Chance ou de Blaise Cendrars, est donc aussi une pensée de la déviance. Peut-être le plagiat, dans son sens large, est-il un geste dialogique, une scénographie de la parole, ouverte à sa propre vacuité et à ses possibilités d'accueil. Mais, chacune à sa manière, les cultures, elles-mêmes entrelacées, imposent leur vision du propre et de l'impropre. Le nihilisme, à la manière de Giorgio Agamben, n'est alors en fin de compte qu'une forme d'appropriation des différences. 